\title{
DSpace@MIT
}

\author{
MIT Open Access Articles
}

\section{Effects of Added Vegetation on Sand Bar Stability and Stream Hydrodynamics}

The MIT Faculty has made this article openly available. Please share how this access benefits you. Your story matters.

Citation: Rominger, Jeffrey T., Anne F. Lightbody, and Heidi M. Nepf. “Effects of Added Vegetation on Sand Bar Stability and Stream Hydrodynamics." Journal of Hydraulic Engineering 136.12 (2010): 994.

As Published: http://dx.doi.org/10.1061/(asce)hy.1943-7900.0000215

Publisher: American Society of Civil Engineers

Persistent URL: http://hdl.handle.net/1721.1/68006

Version: Author's final manuscript: final author's manuscript post peer review, without publisher's formatting or copy editing

Terms of use: Creative Commons Attribution-Noncommercial-Share Alike 3.0 


\section{The Effects of Added Vegetation on Sand Bar Stability}

and Stream Hydrodynamics

3 Jeffrey T. Rominger ${ }^{1}$, Anne F. Lightbody ${ }^{2}$, Heidi M. Nepf ${ }^{3}$

4

5 Abstract: Vegetation was added to a fully-developed sandy point bar in the meander of a

6 constructed stream. Significant changes in the flow structure and bed topography were observed.

7 As expected, the addition of vegetative resistance decreased the depth-averaged streamwise

8 velocity over the bar and increased it in the open region. In addition, the secondary circulation

9 increased in strength, but became confined to the deepest section of the channel. Over the point

10 bar, the secondary flow was entirely outwards, i.e. towards the outer bank. The changes in flow

11 led to changes in bar shape. Although the region of the bar closest to the inner bank accumulated

12 sediment, erosion of the bar and the removal of plants by scouring was observed at the interface

13 between the planted bar and the open channel.

\section{Subject Headings:}

15 Meandering Streams; Secondary Flow; Stream Restoration; Riverine Bars; Riparian Vegetation;

16 Riverbank Erosion; Sediment Transport

Introduction

The most obvious hydraulic impact of aquatic and riparian vegetation is an increase in

20 flow resistance and a reduction in conveyance capacity (Kouwen and Unny 1973; Kouwen,

21 1990; Wu et al 1999). Historically, many channels have been straightened and denuded of

21

${ }^{1}$ Corresponding Author, Department of Civil and Environmental Engineering, Massachusetts Institute of Technology, 77 Massachusetts Ave., Bldg. 48-114, Cambridge, MA 02139, USA, email: jtr@mit.edu

${ }^{2}$ St. Anthony Falls Laboratory, University of Minnesota, 2 Third Ave. SE, Minneapolis, MN 55414, USA

${ }^{3}$ Department of Civil and Environmental Engineering, Massachusetts Institute of Technology, 77 Massachusetts Ave., Bldg. 48-216D, Cambridge, MA 02139, USA 
22 vegetation to accelerate the passage of peak flows, but these anthropogenic modifications can

23 have unintended consequences on water quality, channel stability and stream ecology. For

24 example, many studies have shown that aquatic macrophytes have a positive influence on water

25 quality by utilizing nutrients, producing oxygen and detaining heavy metals and other

26 contaminants (e.g. Chambers and Prepas 1994; Kadlec and Knight 1996; Windham 2003).

27 Vegetation also promotes biodiversity by creating diverse habitats through spatial heterogeneity

28 in the flow (Kemp et al 2000; Crowder and Diplas 2000, 2002).

29 Vegetation is also an important ecosystem engineer within channel systems. By reducing

30 near-bed velocity, in-stream and floodplain vegetation can both reduce erosion and promote

31 deposition. Elliott (2000) explains how the aboveground portion of biomass helps increase

32 sedimentation both by reducing the local flow velocities and by providing additional horizontal

33 surface per volume upon which sedimentation can occur. The presence of vegetation can also

34 exert control over river planform (Tal and Paola 2007). Therefore, widespread planting of

35 vegetation is often advocated as a restoration technique for its ability to increase channel stability

36 and for its ability to remove nutrients from the water (Mars et al. 1999; Abernethy and

37 Rutherford 1998; Simon and Collison 2002; Pollen and Simon 2005). While many studies

38 describe the stabilizing effects of vegetation, few have considered, few have considered the

39 relative contributions of the aboveground biomass and the belowground root system. Corenblit

40 et al. (2007) summarizes several experimental studies that indicate that the aboveground biomass

41 in grasses and other channel-lining vegetation is more important than belowground biomass for

42 sediment stability (Gyssels et al. 2005, James et al. 2002, Prosser et al. 1995). These studies

43 suggest the reduction in erosion observed within regions of vegetation is due mostly to the

44 reduction in the turbulent shear stress at the bed, but acknowledge that the root system remains 
less well understood.

Recent studies have suggested that the addition of vegetation may create regions of

47 enhanced erosion potential. McBride et al. (2007) observed that the presence of vegetation on a

48 floodplain could elevate the turbulence level at the floodplain-channel interface, relative to

49 unvegetated floodplains. They suggested that the presence of the vegetation created a region of

50 high erosion potential at the channel-floodplain interface. Similarly, Temmerman et al. (2007)

51 found the presence of vegetation to concentrate flow and encourage localized erosion in the

52 growth of channels on tidal flats. Bouma et al (2007) created islands of bamboo shoots on an

53 intertidal flat. Over two-years of monitoring they observed deposition to occur within and

54 downstream of the patch, but erosion occurred at the front and sides of the patches. The

55 observations of Bouma et al. (2007) can be related to previous observations around bridge piers.

56 Bridge piers and abutments generally destabilize the substrate around them, resulting in scour

57 holes. Many studies have recorded both the erosion caused by isolated pilings (Melville 1997,

58 Melville \& Chiew 1999), as well as the more complex scouring patterns caused by groups of

59 piles (Ataie-Ashtiani \& Beheshti 2006, Bateni \& Jeng 2007). This area of research confirms that

60 there is a real possibility for in-stream objects, including vegetation, to promote localized

61 erosion. A finite patch of vegetation may operate in a similar fashion. The divergence of flow

62 away from the region of high-drag within the patch results in accelerated flow at the edges of the

63 patch, which in turn creates the regions of erosion observed by Temmerman et al. (2007) and

64 Bouma et al. (2007).

65 To have successful replanting and restoration of channels, we need to understand how the

66 placement and planting density impact the local flow field, which predicts the potential for

67 deposition and erosion near the restored vegetation. This study seeks to provide some insight 
68 into how the addition of vegetation at a specific location on the point bar in a channel bend,

69 alters the flow field, which in turn leads to changes the bed topography.

71 Description of Facility

72 The experiments were conducted in the Outdoor StreamLab (OSL), an experimental

73 facility built on a retired spillway adjacent to the University of Minnesota's St. Anthony Falls

74 Laboratory in downtown Minneapolis. During 2008, within the facility's 40-m by 20-m

75 Riparian Basin, a sand-bed stream was constructed with three meander bends that have an

76 average wavelength of $25 \mathrm{~m}$ and a sinuosity of 1.3 (Figure 1). In the straight sections in between

77 the meanders, riffles were constructed with coarse-grained sediment and cobbles to mimic the

78 pool-riffle geometry of many natural streams. A concrete headbox at the mouth of the stream

79 was supplied with water from the Mississippi River via two 18 in. diameter steel pipes, which

80 were controlled manually with knife-valves. The base flow rate for the stream was $38 \pm 5 \mathrm{~L} / \mathrm{s}$ and

81 was calculated from the height of water above a contracted weir at the upstream end of the

82 stream using an air ultrasonic transducer (Massa Products Corporation, Hingham, MA).

83 The banks of the channel were fixed in geometry and position with coconut fiber matting,

84 but the bed of the channel was mobile, and consisted of coarse-grained sand (median grain size:

$85 D_{50}=0.7 \mathrm{~mm}$ ). A sediment recirculation system carried bedload sediment collected from the

86 downstream end back to the upstream end of the channel using an adjustable rate auger. During

87 the first flood event, point bars formed from the mobile bed material near the inner bank of the

88 second and third meander bends (see Figure 2). These point bars formed within the first few

89 hours of the first flood event on July 10, 2008, and remained as roughly stable artifacts in the

90 stream during the base flow and subsequent flood events in July (Figure 3). The magnitudes of 
91 the water surface slope, derived from the survey data, were $S=0.006$ and 0.007 for the flood

92 level and base level flows, respectively. The magnitude of the average bed slope was 0.007 .

\section{Stream Coordinate System and Momentum Equations}

94 It is useful to define a streamline coordinate system that follows the curvature of the

95 stream, with the downstream coordinate tangent to the stream centerline. This coordinate system

96 is left-handed, orthogonal and curvilinear, similar to systems defined by Smith and McLean

97 (1984) and Dietrich and Smith (1983) and consists of an $s$-axis, tangent to the centerline of the

98 stream and positive in the downstream direction, an $n$-axis, perpendicular to the stream centerline

99 and positive towards the right bank, and a vertical axis, $z$, positive in the upwards direction with $z$

$100=0$ at the water surface. The time-average velocity field is denoted $(u, v, w)$ in the directions $(s$,

$101 n, z)$, respectively. A depth average is denoted by a bracket. The force balance equations in the

102 downstream and cross-stream directions are then:

103

$\left(\tau_{z s}\right)_{b}=-\frac{\rho g h}{(1+N)} \frac{\partial \eta}{\partial s}-\rho \frac{1}{1+N} \frac{\partial}{\partial s}\left\langle u^{2}\right\rangle h-\rho \frac{\partial}{\partial n}\langle u v\rangle h-2 \rho \frac{\langle u v\rangle h}{(1+N) R}-\frac{1}{2} \rho C_{D} a h|\langle u\rangle|\langle u\rangle$

(A1)

(A2)

(A3)

(A4)

(A5)

(A6)

104

$-\left(\tau_{z n}\right)_{b}=\rho g h \frac{\partial \eta}{\partial n}-\rho \frac{\left\langle u^{2}\right\rangle h}{(1+N) R}+\rho \frac{1}{1+N} \frac{\partial}{\partial s}\langle u v\rangle h+\rho \frac{\partial}{\partial n}\left\langle v^{2}\right\rangle h+\rho \frac{\left\langle v^{2}\right\rangle h}{(1+N) R}-\frac{1}{2} C_{D} a h \mid\langle v\rangle\langle v\rangle$

(B3)

(B4)

(B5)

(B6)

(B7)

105 Terms A1 and B1 represent the boundary shear stress, $\eta$ is the super-elevation of the water

106 surface, $h$ is the total depth of the water column, $\rho$ is the density of water, $g$ is the acceleration

107 due to gravity, $R$ is the local radius of curvature of the stream and the non-dimensional

108 coordinate $N=n / R$. Terms A6 and B7 represent the additional hydraulic resistance provided by

109 vegetation, when present. The vegetation density is represented by the frontal area per unit

110 volume, $a$, and $C_{D}$ is the vegetation drag coefficient.

111 To the leading order, the dominant terms in the cross-stream force balance are often B2 
112 and B3, the cross-stream pressure gradient and the centrifugal force, respectively. Near the

113 bottom of the water column, bed friction causes a lower velocity, and thus a lower centrifugal

114 force. The pressure gradient, which is uniform over depth, is thus unbalanced near the bed,

115 driving a secondary flow toward the inside of the meander, i.e. toward the point bar. Near the

116 water surface, the velocity is higher than the depth-average, and the centrifugal force exceeds the

117 pressure gradient, causing the secondary flow to be outward, away from the point bar. In this

118 paper we examine how the addition of vegetation to a point bar changes this secondary

119 circulation, and consider the effects this has on water and sediment supply to the sand bar, as

120 well as the resulting changes in bed topography.

\section{Experimental Methods}

123 Bank-full flood events, representative of the average flood magnitude in natural channels,

124 were created at approximately weekly intervals. Each flood lasted 9 hours and had a constant

125 flow rate of $208 \pm 5 \mathrm{~L} / \mathrm{s}$. At the start of each of the experimental floods, the flow rate was

126 gradually increased from the base flow rate $(38 \mathrm{~L} / \mathrm{s})$ to the bank-full level $(208 \mathrm{~L} / \mathrm{s})$ over a period

127 of ten minutes. The flow rate in the stream was monitored continuously and adjusted manually

128 so as to be independent of changes in the flow in the Mississippi River. Velocity measurements

129 were made during each of the repeated 9-hour, bank-full flood events. A 3D, 4-beam, sideways-

130 looking, fixed-probe, laboratory Nortek Vectrino Acoustic Doppler Velocimeter (ADV), was

131 used to simultaneously measure velocity in the $s, n$ and $z(u, v$ and $w)$ directions at different cross

132 sections along the length of the stream. The ADV was mounted on a motorized traverse oriented

133 perpendicular to the local stream direction, i.e. along the local $n$-axis. At each cross section,

134 velocity was measured at roughly ten points in the horizontal, $n$ - axis, and a varying number of 
135 points in the vertical, $z$-axis, due to the varying depth. The highest data points were within two 136 centimeters of the surface, and the lowest points were within $5 \mathrm{~cm}$ of the bed. The velocity was 137 recorded at each point for between 120 to 240 seconds at $25 \mathrm{~Hz}$. The velocity data was filtered 138 to exclude erroneous values with low correlation coefficients or low signal-to-noise ratios 139 (SNRs). The most common cause of erroneous data points was an obstruction in the sampling 140 volume such as stream debris or a solid surface like the streambed or bank. Each transect was 141 aligned perpendicularly to the streambanks, and the transect position was marked with stakes and 142 surveyed to ensure repeatable positioning throughout the summer. Alignment was ensured by 143 checking the total flow-rate at each transect against the flow-rate delivered from the upstream 144 headbox.

145 Two types of tracer tests, using Rhodamine WT, provided information regarding the 146 transport at the reach-scale as well as locally around the point bar. First, to measure reach147 averaged parameters, dye was injected as a line source near the mouth of the stream and a 148 fluorometer was set up near the downstream bridge (Figure 1). We diluted $2 \mathrm{ml}$ of Rhodamine 149 WT into a $500 \mathrm{ml}$ solution and injected the solution over the stream's cross-section over a period 150 of less than 1 second (approximately instantaneous). A submersible recording fluorometer

151 (SCUFA, Turner Designs) recorded dye concentrations in the water at the downstream bridge at 152 a rate of $1 \mathrm{~Hz}$. To estimate the reach-scale longitudinal dispersion and the retention time, the 153 downstream concentration records were analyzed using the method of moments (e.g. Murphy et 154 al., 2007). Second, to estimate the difference in transport time-scales between the vegetated and 155 unvegetated regions in the second meander, an identical mixture of dye was injected 156 instantaneously as a planar source near the mouth of the stream and fluorometers were set up in 157 Planes C and D (Figure 1). The SCUFA was set at mid-depth in the middle of the vegetated sand 
158 bar (points C1 and D1), and a Seapoint Sensors Fluorometer, sampling at $7.5 \mathrm{~Hz}$, was set up in

159 the same plane at the midpoint of the open region (points C2 and D2 in Figure 1). The two

160 fluorometers were synchronized using a stopwatch.

161 Floods were started on July 10, 2008 and a steady bathymetry was established during the

162 first flood. Surveys made with a Leica Total Station were used to record channel geometry as it

163 developed over the summer. On August 5, 2008, two reed species, Juncus effusus and Scirpus

164 atrovirens were planted on the portion of the sand bar in the second meander that was exposed at

165 base flow. This vegetation was planted in a uniform, staggered array that produced a vegetated

166 frontal area per unit volume of $a=5.2 \mathrm{~m}^{-1}$, where $a=n_{\text {plant }} d_{\text {plant }}, n_{\text {plant }}=69 \mathrm{~m}^{-2}$ is the number of

167 plant plugs per unit area, and $d_{\text {plant (avg) }}=.075 \mathrm{~m}$ is the characteristic diameter of a single plant

168 plug (a close grouping of several individual stems. See Figure 4). Throughout the subsequent

169 floods, the velocities and the reach-scale transport parameters were monitored for changes using

170 the methods described above.

171

172 Results and Discussion

173 As expected, a secondary circulation was observed in the meander bends prior to the

174 addition of vegetation. This circulation was most intense near the apex of the meander (Plane B

175 of Figure 1), with a strong lateral outflow near the water surface and a return current near the bed

176 of the stream (Figure 5b). The secondary circulation predominantly occupied the deeper part of

177 the cross section, with smaller lateral, $v$, and vertical velocities, $w$, over the point bar. The depth-

178 averaged streamwise velocity, $\langle u\rangle$, was highest near the outer bank of the meander and smallest

179 over the point bar (Figure 5a).

180 After nearly one month of flow, including five floods, the point bar in the second 
181 meander was planted with emergent vegetation during base flow conditions. During the first

182 flood event after the planting (August 6, 2008), the cross-sectional geometry changed rapidly due

183 to the flow disturbance created by the plants (Figure 3). The outermost row of plants was

184 scoured away, as well as part of the next outermost row, removing approximately $50 \mathrm{~cm}$ of the

185 emergent point bar's width along with most of the vegetation in this zone. This loss of sediment

186 and resulting loss of point bar area, observed in the early stages of the flood, was confirmed by

187 photographic and survey data. Similar measurements for the unvegetated point bar in meander 3

188 showed no loss in emergent bar area, confirming that the loss observed in the second point bar

189 was due to the added vegetation. The plants and bar area that were not scoured away in the first

190 hours of the first flood were stable for the remainder of the summer flood sequence.

191 Both the depth-averaged streamwise velocity and the secondary circulation at the apex of

192 meander 2 changed significantly after the vegetation was added (Figure 6). First, the depth-

193 averaged streamwise velocity decreased over the bar and increased in the open region (Figure

194 6a). Second, the secondary circulation increased in strength, but was confined to the deepest

195 section of the channel. The depth-averaged centrifugal force, i.e. term B3 (e.g. as in Kitanidis

196 and Kennedy, 1983), which drives the secondary circulation, increased by $30 \%$ after the addition

197 of vegetation to the bar (Figure 7). A direct calculation of the average $v$-component of the

198 velocity at the apex of the bend, calculated by taking the root-mean-square of the velocities at

199 each cross-stream coordinate in the channel, showed approximately a $50 \%$ increase after the

200 vegetation was added. Because the strength of the secondary circulation increased significantly

201 and the flow at the outer bank was enhanced, erosion would be likely in a natural channel, and

202 this could accelerate the meander growth. This could not occur in our channel because the banks

203 were fixed in position by buried fiber matting. Finally, over the point bar, a strong outwards 
204 (toward the outer bank) flow extended over the entire depth of the water column.

205 The difference in the velocity field before and after the insertion of vegetation occurred

206 because the vegetation increased the hydraulic resistance over the point bar. Defining the bed

207 stress by a bed drag coefficient, $\left(\tau_{z s}\right)_{b}=-\rho C_{f} \mid\langle u\rangle\langle u\rangle$, we can compare the hydraulic resistance

208 provided by the bed (term A1) with that provided by the vegetation (term A6), by comparing the

209 terms $C_{f}(\approx .002$, for a sand bed $)$ and $C_{D} a h\left(\approx 0.8\right.$, assuming $\left.C_{D}=\mathrm{O}(1)\right)$. This comparison

210 indicates that the addition of vegetative drag (term A6) increased the total drag on the bar by two

211 orders of magnitude, significantly retarding the flow and causing a lateral diversion toward the

212 open channel. As the flow was diverted away from the region of high drag, the downstream

213 velocity accelerated near the edge of the vegetation, causing the observed scour. Specifically,

214 the velocity at the vegetation edge $(y=50 \mathrm{~cm})$ increased from $45 \mathrm{~cm} / \mathrm{s}$ before the addition of

215 vegetation (Figure $5 \mathrm{a}$ ) to $55 \mathrm{~cm} / \mathrm{s}$ after the addition of vegetation (Figure $6 \mathrm{a}$ ).

216 These changes in the secondary circulation can be explained by consideration of the

217 spatial acceleration terms in the cross-stream momentum balance. As water decelerated over the

218 point bar, an effect that was magnified by the presence of vegetation, the downstream slope of

219 the water surface was reduced. A concomitant acceleration of the flow in the deeper portion of

220 the channel increased the downstream slope in this region of flow. The combined effect of these

221 changes in the downstream surface slope was a reduction in the cross-stream surface slope (i.e.

222 the cross stream pressure gradient) near the apex. This phenomenon can also be observed where

223 flow shoals over a bare point bar, but here it was greatly magnified by the two orders of

224 magnitude increase in resistance introduced with the vegetation. For a further discussion of the

225 effects of spatial accelerations on the surface slope and a very helpful diagram, see Dietrich and

226 Smith (1983). The result is that after the vegetation was added, the centrifugal force exceeded 
227 the cross-stream pressure gradient over the entire depth over point bar, and caused a lateral flow

228 toward the open channel and outer bank that extended over the entire water depth in the

229 vegetated region, i.e. there was no return flow at the bed (Figure 6b). Importantly, the return

230 current near the bed was then limited to only the deepest parts of the channel (Figure 6b), in

231 contrast to the conditions before the vegetation (Figure 5b), in which the return flow extended

232 onto the bar. Importantly, these observations suggest that the addition of vegetation changed the

233 secondary flow in such a way as to cut off sediment supply from the open channel to the bar.

234 Finally, measurements of the bed geometry taken before and after the vegetation was

235 added show how the depth-profile changed (Figures $5 \mathrm{~b}, 6 \mathrm{~b}$ and $8 \mathrm{~b}$ ). Approximately $5 \mathrm{~cm}$ of

236 sediment was deposited within the vegetation and between 0 and $4 \mathrm{~cm}$ of erosion occurred near

237 the edge of the vegetation. Erosion $(0$ to $5 \mathrm{~cm})$ also occurred in the deeper parts of the cross

238 section near the outer bank. The new bed geometry was recorded on August 26, 2008, four

239 floods after the addition of vegetation, but the changes were observed to take place primarily

240 during the first flood following the addition of the vegetation. The pattern of deposition within

241 the vegetated patch and erosion at its edge is similar to that observed by Bouma et al (2007)

242 within and around a circular patch of bamboo reeds.

244 Implications for Erosion

245 Several studies suggest that plant growth can be inhibited by flow. Chambers et al.

246 (1991), observed a strong negative correlation between macrophyte biomass and current

247 velocities, with little vegetation present above a threshold of $1 \mathrm{~m} / \mathrm{s}$. Similarly, Nilsson (1987)

248 found the percentage of bare ground along a reach increased with increasing current velocities in

249 the free stream. Bouma et al. (2007) saw similar sediment patterns within an artificial patch of 
250 vegetation with erosion near the edges and deposition deeper within the patch. These studies

251 imply that particular planting strategies can be less successful if they lead to locally enhanced

252 velocities. To gain insight into this problem, we consider whether the observed loss of

253 vegetation introduced in our study is consistent with our physical understanding of plant and

254 sediment stability.

255 There are two physical mechanisms that limit the invasion and propagation of vegetation

256 into a stream channel. First, for a given unconsolidated sandy bed, the substrate becomes mobile

257 above a certain bed shear stress, defined by the Shields Parameter. But sediment motion alone

258 does not govern the presence of plant life. A plant may be able to survive an area with weak

259 sediment motion, but a rapidly scouring bed will cause plants to uproot and will preclude the

260 growth of aquatic vegetation that depends on the substrate for stability (Fonseca et al., 1983).

261 Second, plants have an inherent lodging velocity that defines the flow speed at which the plant

262 material fails. This value is a function of the stem flexural stiffness, geometry and natural

263 roughness of the plant (Duan et al. 2002). For the mobile sand bed found in the OSL, the

264 scouring threshold appeared to be reached before the lodging velocity because the plants lost

265 during the flood came out as intact plugs, with no observed damage to the plant material. This

266 implies that the plants dislodged because the substrate around them eroded, so that to understand

267 this loss we must consider the changes in sediment stability.

268 The Shields Parameter, $\psi$, describes the ratio of destabilizing (drag) and stabilizing

269 forces (settling) for cohesionless sediment. This parameter is defined as

$$
\psi=\frac{\tau_{b}}{\left(\rho_{s}-\rho\right) g d}=\frac{\rho C_{f}\langle u\rangle^{2}}{\left(\rho_{s}-\rho\right) g d}
$$

271 Here, $\rho_{s}$ is the sediment density, $C_{f}$ is the coefficient of friction of the bed and $d$ is the sediment

272 grain diameter. We can evaluate the changing stability of the bed by comparing the Shields 
273 Parameter before and after the vegetation was added. Because the sediment is unchanged, we

274 can assume that both the settling forces and the bed friction coefficient do not change. It is then

275 convenient to form the following ratio, to describe the changes in bed stability:

$$
\frac{\psi_{\text {veg. }}}{\psi_{\text {unveg. }}}=\frac{\left\langle u_{\text {veg. }}\right\rangle^{2}}{\left\langle u_{\text {unveg. }}\right\rangle^{2}}
$$

277 The areas in the vegetated cross section where erosion was observed corresponded to a Shields

278 Parameter ratio of greater than 1 (Figure 8). Similarly, the areas in which deposition occurred

279 corresponded to a Shields Parameter ratio of less than 1. The correspondence between the

280 Shields Parameter ratio and the observed erosion/deposition patterns suggests that the vegetation

281 changed the stability of the bed by altering the local flow speed and thus the local bed stress.

282 This is consistent with the observation above, that the plants lost were removed intact, i.e. the

283 sediment eroded away around the plug. Further, this set of experiments reinforces the theory that

284 high flow energy and rapid sediment scour can preclude vegetative growth and propagation. The

285 recorded changes in the bed profile agree well with the areas where vegetation was lost from the

286 planted array. It is important to note that this ratio of the Shields Parameters indicates tendencies

287 only. It does not suggest that certain areas will erode indefinitely and other areas will continue to 288 accrete.

289 A more developed root system may have better anchored the plants in the sediment, but 290 research shows that erosion of the sediment near the edge of the vegetation is representative of 291 the behavior in real systems. In Bouma et al. (2007), the added vegetation was anchored $30 \mathrm{~cm}$ 292 into the sediment, and despite the fact that none of the plants were lost during the experiment, 293 significant erosion was still observed near the edges of the artificial patch. This indicates that the 
294 diversion of flow would have caused similar erosion of the sediment whether the plants were 295 able to remain anchored or not.

296 Furthermore, in this set of experiments the addition of vegetation to the stream channel is

297 not directly analogous to a natural scenario where locally present vegetation slowly colonizes the

298 bank via hydrochory or other processes. This experiment more closely represents a restoration

299 scenario, where vegetation is added to an emergent point bar and the resulting adjustments

300 caused by bank-full flow are observed, measured and documented. These experiments would be

301 similar to a scenario in which vegetation propagated into a channel during an extended period of

302 low flow, then was forced to adjust upon the return of bank-full flow levels, potentially showing

303 similar patterns of erosion and deposition of suspended sediment.

Pathway of Surface Water over the Vegetated Bar

The availability of suspended sediment to the bar, as well as the water quality on the bar,

307 both depend upon the supply of new water to this region. The tracer measurements made near

308 the vegetated bar allowed us to draw conclusions about the advective and diffusive transport near

309 the bar. Figure 9 shows the residence time distributions at two longitudinal positions for dye

310 passing through the vegetation (fluorometer positions C1 and D1 in Figure 1) and dye passing

311 through the adjacent open channel (fluorometer positions C2 and D2 in Figure 1). The arrival

312 time of the peak dye concentration is delayed in the vegetation compared to the arrival time of

313 the peak concentration in the open channel. The delays observed at cross-sections C and D

314 (Figure 1) are $\Delta T_{\text {Plane } C}=18 \pm 10 \mathrm{sec}$. and $\Delta T_{\text {Plane } D}=27 \pm 3 \mathrm{sec}$. If the vegetated region was a

315 distinct advection zone, with little lateral diffusive exchange between the open channel and the

316 vegetated region, then the delay in the dye passage, $\Delta T_{\text {Plane } C}$, should be approximately two thirds 
317 of $\Delta T_{\text {Plane } D}$, because Plane $\mathrm{C}$ is $2 / 3$ the distance between Plane A (leading edge of the vegetation)

318 and Plane D. Assuming the velocities remained close to constant, the observed timescales

319 agreed with this hypothesis, suggesting that dye entered the sand bar at the upstream edge and

320 advected in streamlines roughly parallel to the bank with little lateral supply from the open

321 channel. Further, we can estimate the lateral diffusivity, $D$, from photographs of the dye

322 evolution along the channel (e.g. as in Nappo and Hiscox, 2008). Then, using the width of the

323 vegetation, $b=0.7 \mathrm{~m}$, the lateral diffusive velocity, $D / b=0.004 \mathrm{~m} / \mathrm{s}$, was found to be much

324 smaller than the measured lateral velocity, $v=-0.12 \mathrm{~m} / \mathrm{s}$. Although some mixing was observed

325 near the edge of the vegetation, the turbulent diffusion was not large enough to offset the

326 significant outward lateral advection, such that diffusion provides a negligible scalar flux to the

327 region of water over the bar. Therefore, longitudinal advection from the upstream portion of the

328 vegetated sand bar was the dominant source of channel water to the region above the bar, and the

329 only potential sediment supply as well. These findings were confirmed by photographs of dye

330 streamlines within the vegetation, which showed little lateral mixing across the boundary (See

331 Figure 10).

332 The reach-scale tracer tests indicated that the longitudinal dispersion coefficient $\left(K_{x}=5.1\right.$

$333 \pm 0.2 \times 10^{-4} \mathrm{~m}^{2} \mathrm{~s}^{-1}$ ) did not change (within uncertainty) after the addition of vegetation on the bar.

334 There was also no significant change in the residence time distribution following the addition of

335 the vegetation. Although the vegetated sand bar creates a large slow-zone, only about $10 \%$ of

336 the flow encounters this region, with the remaining flow diverted around it. In order to observe

337 an impact on reach-scale dispersion, the flow needs to encounter multiple slow-zones, so that a

338 larger fraction of the total flow experiences at least one such zone. 


\section{Conclusions}

Vegetation was added to a fully-developed sandy point bar near the convex bank of a

342 stream meander. Both the flow field and the bed topography changed dramatically after the

343 addition of the vegetation. Before the vegetation was added, the secondary circulation included

344 the shallow areas above the sand bar, with the return current near the bed acting as a supply of

345 surface water and sediment to the bar region. After the addition of vegetation, the secondary

346 circulation was present only in the deepest section of the meander, near the outer bank, and the

347 flow above the sand bar was outward over the entire water column. Importantly, the vegetation

348 altered the secondary circulation sufficiently to cut off a source of water and sediment to the bar.

349 Deposition occurred within the patch of vegetation, near the inner stream bank,

350 illustrating the positive feedback through which vegetation can stabilize landforms (e.g. Tal and

351 Paola 2007). However, Erosion occurred near the lateral edge of the vegetation, resulting in a

$35230 \%$ loss of emergent bar width at the apex at base flow. This included the removal of some

353 newly planted vegetation. The positions of erosion and deposition were consistent with the

354 observed changes in the Shields Parameter. Areas where the Shields Parameters ratio was

355 greater than unity corresponded to regions of erosion and areas where the ratio was less than

356 unity corresponded to areas of deposition. The observations suggest that the spatial accelerations

357 caused by the presence of the vegetation shifted the sand bar area to a new geometric

358 equilibrium. More generally, these observations exemplify why the edges of a vegetation patch

359 can be regions of enhanced erosion, as was observed previously by Bouma et al (2007) and

360 Temmerman et al. (2007). Specifically, flow divergence associated with the additional

361 vegetative-drag results in flow accelerations at the patch edge, which can lead to erosion at the

362 patch edge. This phenomenon is similar to the scour patterns observed in the field around 
363 individual flow obstructions, such as bridge piers or abutments.

\section{Acknowledgements}

This material is based upon work supported by the National Science Foundation under

366 Grant No. EAR 0738352. Any opinions, findings, and conclusions or recommendations

367 expressed in this material are those of the authors and do not necessarily reflect the views of the

368 National Science Foundation. Research assistance was provided by Katie Kramarczuk, June

369 Sayers, Alex Nereson, Johnathan Wacloff, Eric Johnson, and Mary Presnail. This work was also

370 supported by the STC program of the National Science Foundation via the National Center for

371 Earth-surface Dynamics under the agreement Number EAR- 0120914. We are grateful for the

372 insightful comments of two anonymous reviewers which helped to improve the paper.

374 References

375 Abernethy, B. and I.D. Rutherford, 1998. Where along a river's length will vegetation most 376 effectively stabilise stream banks? Geomorphology, 23, 55-75.

377 Ataie-Ashtiani, B. and A.A. Beheshti, 2006. Experimental investigation of clear-water local

378 scour at pile groups. J. Hydr. Eng. 132(10), 1100-1104.

379 Bateni, S.M and D.S. Jeng, 2007. Estimation of pile group scour using adaptive neuro-fuzzy $380 \quad$ approach. Ocean Eng. 34(8-9), 1344-1354.

381 Bouma, T.J. van Duren, L.A., Temmerman, S., Claverie, T., Blanco-Garcia, A., Ysebaert, T., and P.M.J. Herman. Spatial flow and sedimentation patterns within patches of epibenthic structures: combining field, flume and modeling experiments. Cont. Shelf. Res. 27, 10201045. doi:10.1016/j.csr.2005.12.019

385 Chambers, P.A., Prepas, E.E., Hamilton, H.R., and M.L. Bothwell, 1991. Current velocity and 
its effect on aquatic macrophytes in flowing waters. Ecol. Appl., 1(3), 249-257.

387 Crowder, D.W. and P. Diplas, 2000. Using two-dimensional hydrodynamic models at scales of ecological importance. J. of Hydr., 230, 172-191.

389 Crowder, D.W. and P. Diplas, 2002. Vorticity and circulation: spatial metrics for evaluating flow complexity in stream habitats. Can. J. Fish. Aquat. Sci., 59, 633-645.

Dietrich, W.E and J.D. Smith, 1983. Influence of the Point Bar on Flow Through Curved Channels. Water Res. Res., 19(5), 1173-1192.

Duan, J.G., R.H. French and J. Miller, 2002. The lodging velocity for emergent aquatic plants in open channels. J. of Amer. Water Res. Assoc., 38(1), 255-263.

Fonseca, M.S. and J.S. Fisher, 1986. A comparison of canopy friction and sediment movement between four species of seagrass with reference to their ecology and restoration. Mar. Ecol. Prog. Ser., 29, 15-22.

Fonseca, M.S., Zieman, J.C. Thayer, G.W., and J.S. Fisher, 1983. The role of current velocity in structuring eelgrass (Zostera marina L.) meadows. Est., Coast. and Shelf Sc., 17, 367380.

401 Kadlec, R.H. and R.L. Knight, 1996. Treatment Wetlands. Lewis Publishers, Boca Raton, FL. 402 Kemp, J., Harper, D., and G. Crosa, 2000. The habitat-scale ecohydraulics of rivers. Ecological $403 \quad$ Engineering, 16,17-29.

404 Kitanidis, P.K. and J.F. Kennedy, 1984. Secondary current and river-meander formation. $J$. Fluid Mech., 144, 217-229.

406 Kouwen, N., 1990, Modern Approach to Design of Grassed Channels, J. Irrigation and Drainage. 118(5), 733-743.

408 Kouwen, N., and T. Unny, 1973, Flexible Roughness in Open Channels. J. Hydraul. Div. 
99(HY5), 713-728.

410 Mars, M., M. Kuruvilla and H. Goen, 1999. The role of submergent macrophyte triglochin 411 huegelii in domestic greywater treatment. Ecol. Eng., 12, 57-66.

412 Melville, B.W., 1997. Pier and abutment scour: integrated approach. J. Hydr. Eng., 123(2), 125$413 \quad 136$.

414 Melville, B.W. and Y. Chiew, 1999. Time scale for local scour at bridge piers. J. Hydr. Eng., 415 125(1), 59-65.

Murphy, E., H. Nepf, M. Ghisalberti, 2007. Longitudinal dispersion in vegetated channels, Water Res. Res., 43, W05438, doi:10.1029/2006WR005229.

Nappo, C.J., Miller D.R., and A.L. Hiscox, 2008. Atmospheric turbulence and diffusion estimates derived from plume image analysis, $15^{\text {th }}$ Joint Conference on the Applications of Air Pollution Meteorology with the A\&WMA. Retrieved February 12, 2009, from http://ams.confex.com/ams/pdfpapers/128980.pdf.

Nilsson, C., 1987. Distribution of stream-edge vegetation along a gradient of current velocity. $J$

424 Pollen, N. and A. Simon, 2005. Estimating the mechanical effects of riparian vegetation on stream bank stability using a fiber bundle model. Water Res. Res., 41, W07025, doi:10.1029/2004WR003801.

Simon, A. and A.J.C. Collison, 2002. Quantifying the mechanical and hydrologic effects of

430 Smith, J.D. and S.R. McLean, 1983. A model for meandering streams. Water Res. Res., 20(9) 1301-1315. 
Tal, M. and C. Paola, 2007. Dynamic single-thread channels maintained by the interaction of flow and vegetation. Geology, 35(4), 347-350.

434 Temmerman, S., Bouma, T.J., Van de Koppel, J., Van der Wal, D., De Vries, M.B., and P.M.J. Herman. Vegetation causes channel erosion in a tidal landscape. Geology, 35(7), 631634. doi: 10.1130/G23502A.1

Windham, L., Weis, J.S., and P. Weis, 2003. Uptake and distribution of metals in two dominant salt marsh macrophytes, Spartina alterniflora (cordgrass) and Phragmites australis (common reed). Estuarine, Coastal and Shelf Science, 56, 63-72. and submerged vegetation. J. Hydr. Eng., 125(9), 934-942.

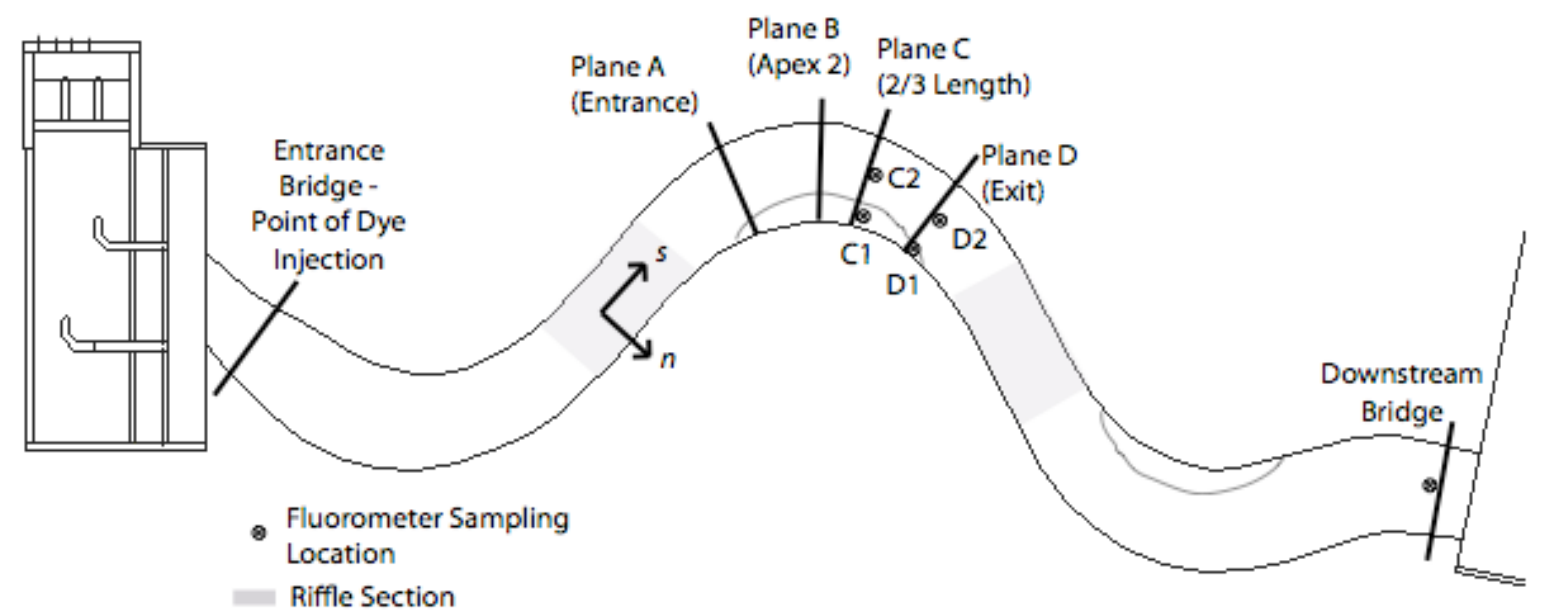

Figure 1: Plan view of the Riparian Basin of the Outdoor Stream Lab indicating measurement locations. 


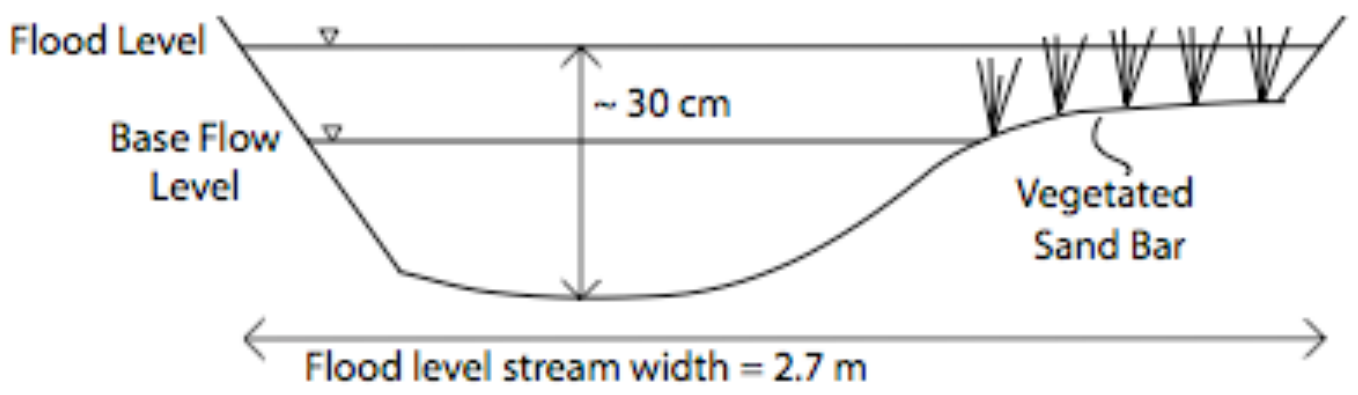

Figure 2: Diagram of the stream cross section with the vegetation added to the area of the sand bar emergent at baseflow. The dimensions provided are the design dimensions and do vary somewhat due to the mobility of the bed. 

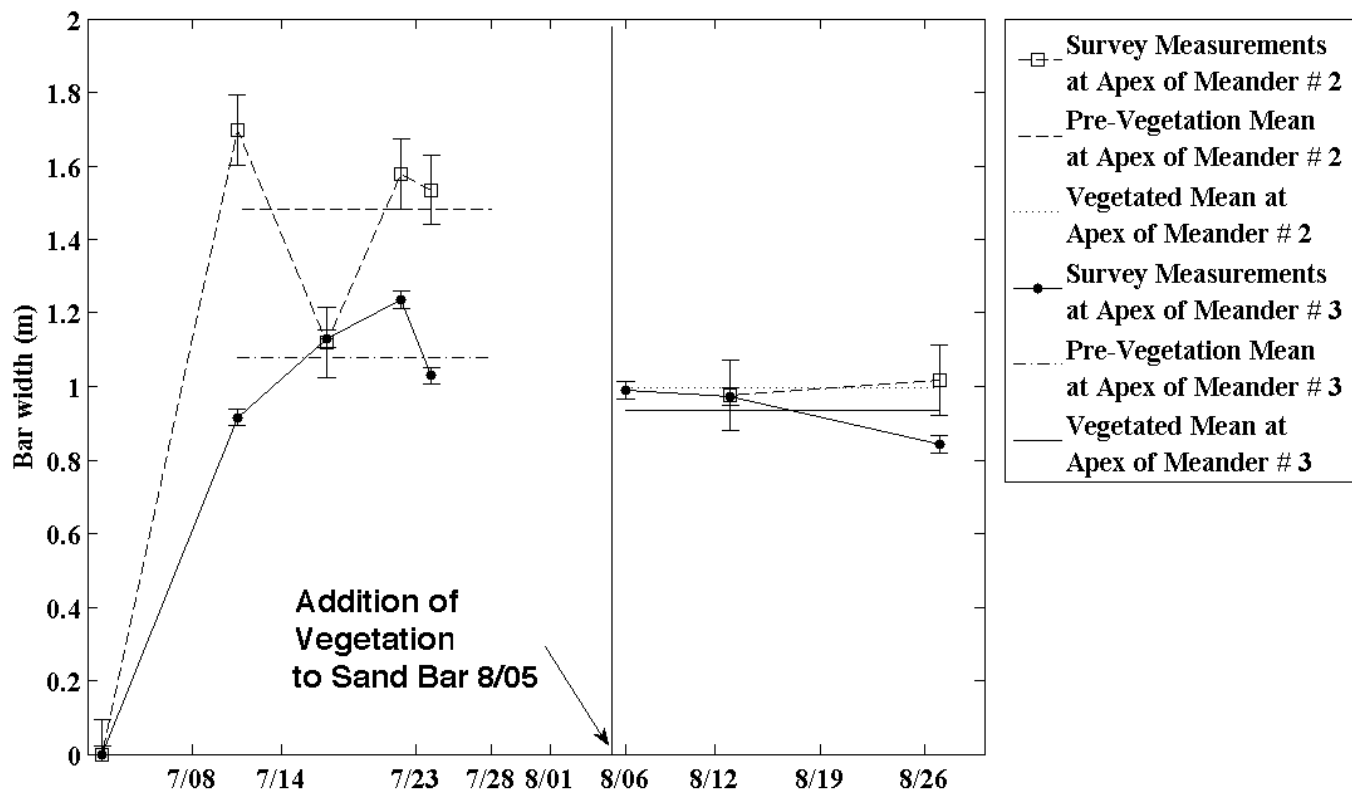

Figure 3: The width of the point bar developed over time, beginning with the first flood event on July 10, 2008, and also with the addition of vegetation to the point bar in Bend \#2 on August 5, 2008. These widths were derived from survey data points taken at the apex of meander 2 . The pre-vegetation mean width is $148 \pm 11 \mathrm{~cm}$. The vegetated mean width is $99 \pm 2 \mathrm{~cm}$. 

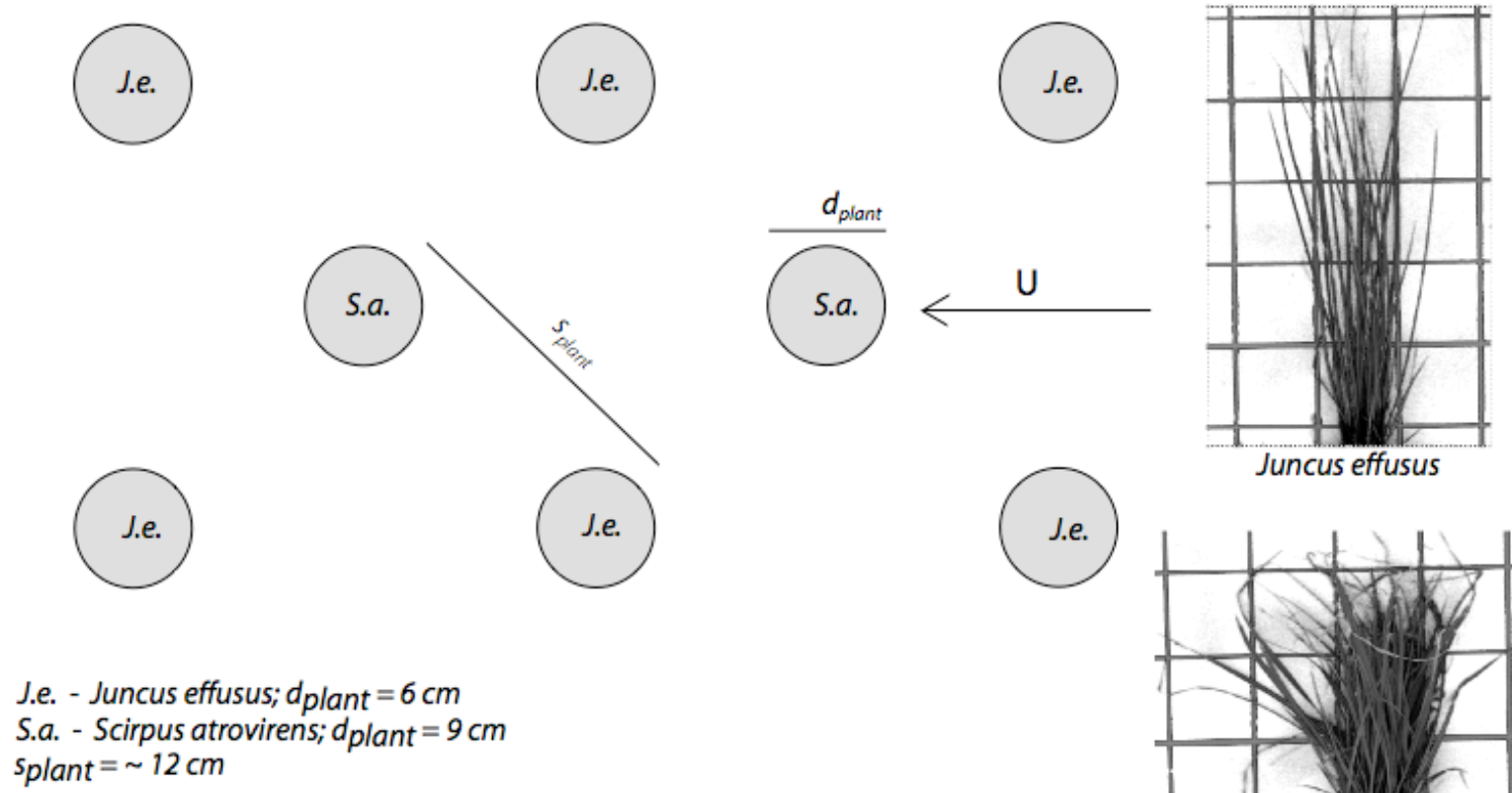

Figure 4: Plan view schematic of the vegetative array on the point bar in meander \#2. The length, $d_{\text {plant }}$, refers to the effective width of the plant (averaged over its height), which is the combined width of all of the stems from a single plug projected onto the streamflow. The spacing, $s_{\text {plant }}$, refers to the average distance between the centers of two plugs. The thumbnail photos show the plant plugs against a $5 \mathrm{~cm}$ grid. 

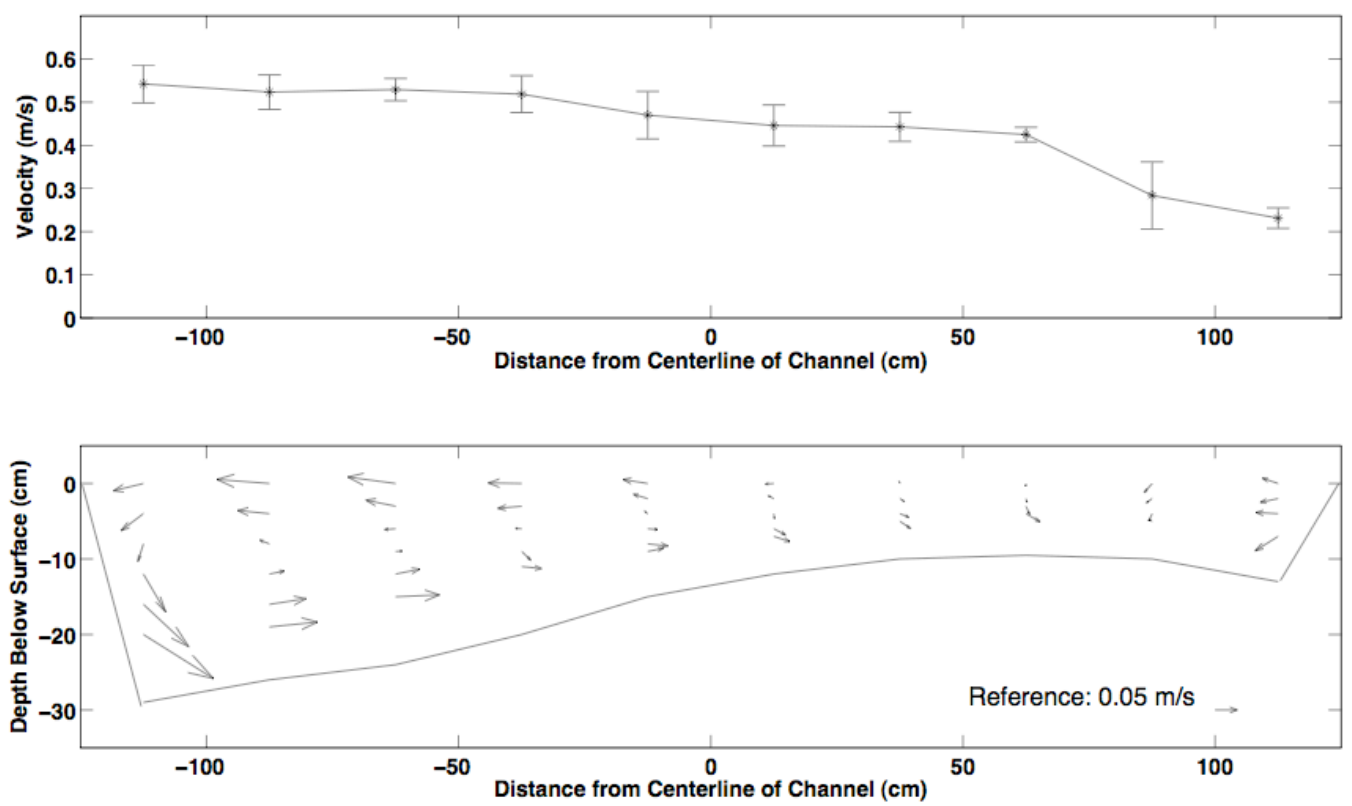

535

536

537

538

539

540

541

542

543

544

545

546

547

FIGURE 5: Velocity Measurements at Apex 2 on July 16, 2008. (a) Depth averaged downstream velocity, $\langle u\rangle$, and (b) velocity components in the lateral and vertical directions, $v$ and $w$, showing the secondary circulation in the $n-z$ plane. The cross-sectional outline shows the measured bed profile, measured by hand from the stream surface. 

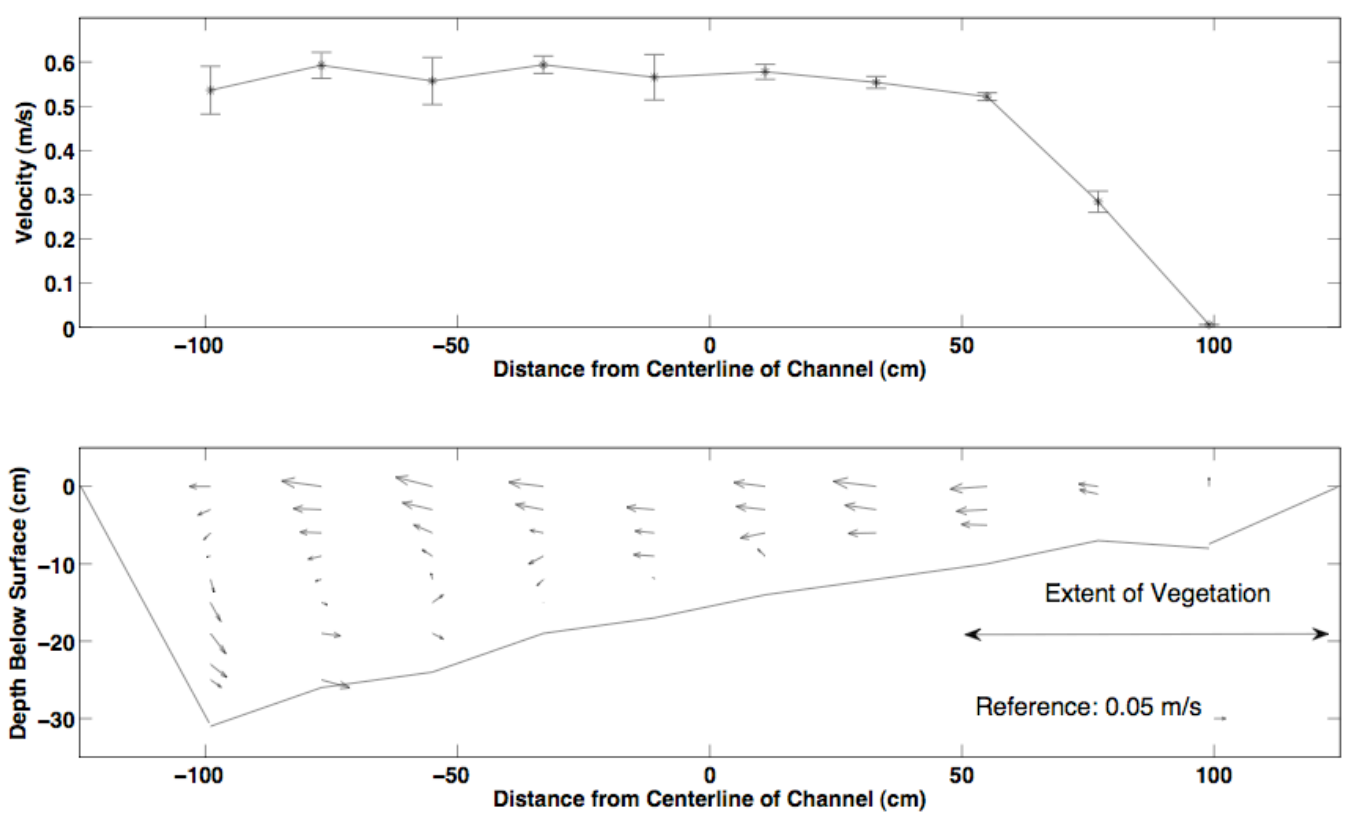

FIGURE 6: Velocity Measurements at Apex 2 with the vegetated sand bar on August 26, 2008. (a) Depth averaged downstream velocity, $\langle u\rangle$, and (b) velocity components in the lateral and vertical directions, $v$ and $w$, showing the secondary circulation in the $n-z$ plane. Note the lateral outflow present over the entire stream depth near the right bank. The cross-sectional outline shows the measured bed profile, measured by hand from the stream surface. 


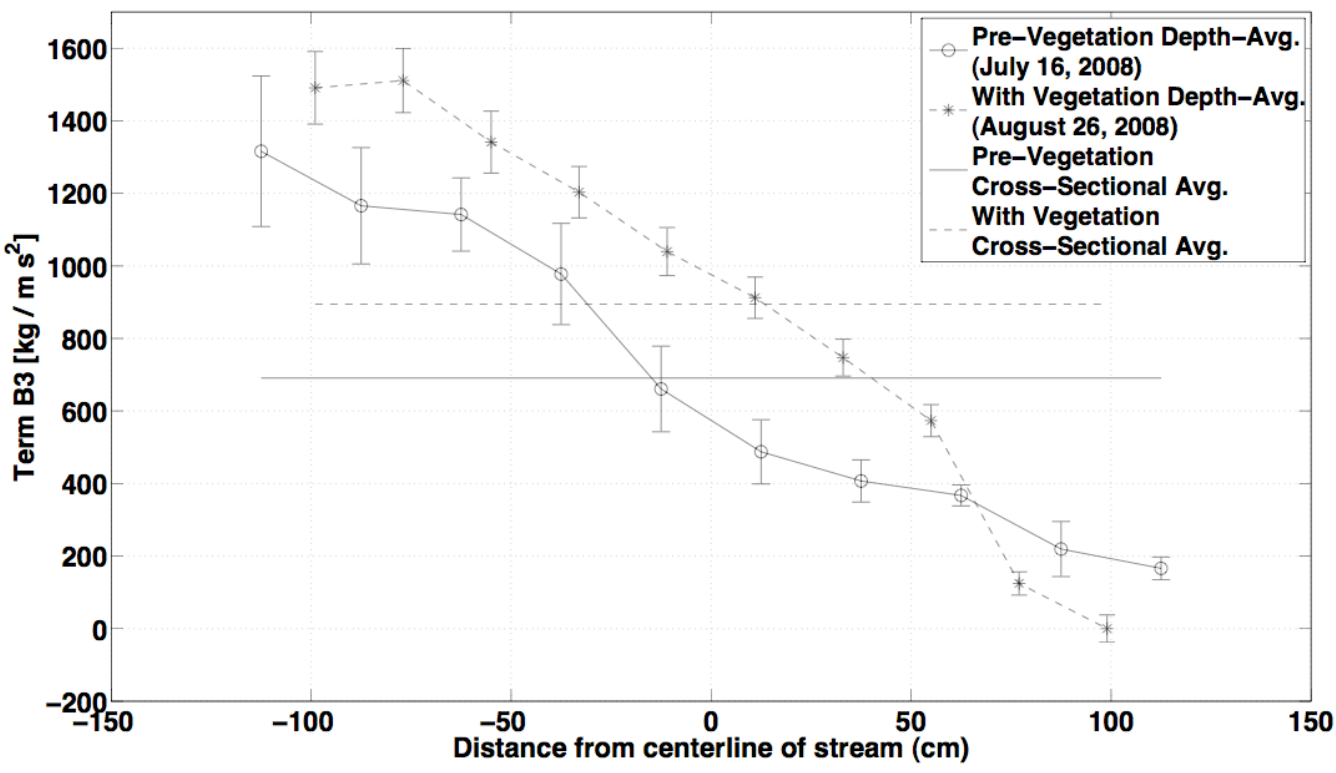

561

562

563 FIGURE 7: The depth averaged centrifugal force and the cross-sectionally averaged centrifugal 564 force at the apex of Meander \# 2 from before and after the vegetation was added to the system.

565

566

567

568

569

570

571

572

573

574

575

576 

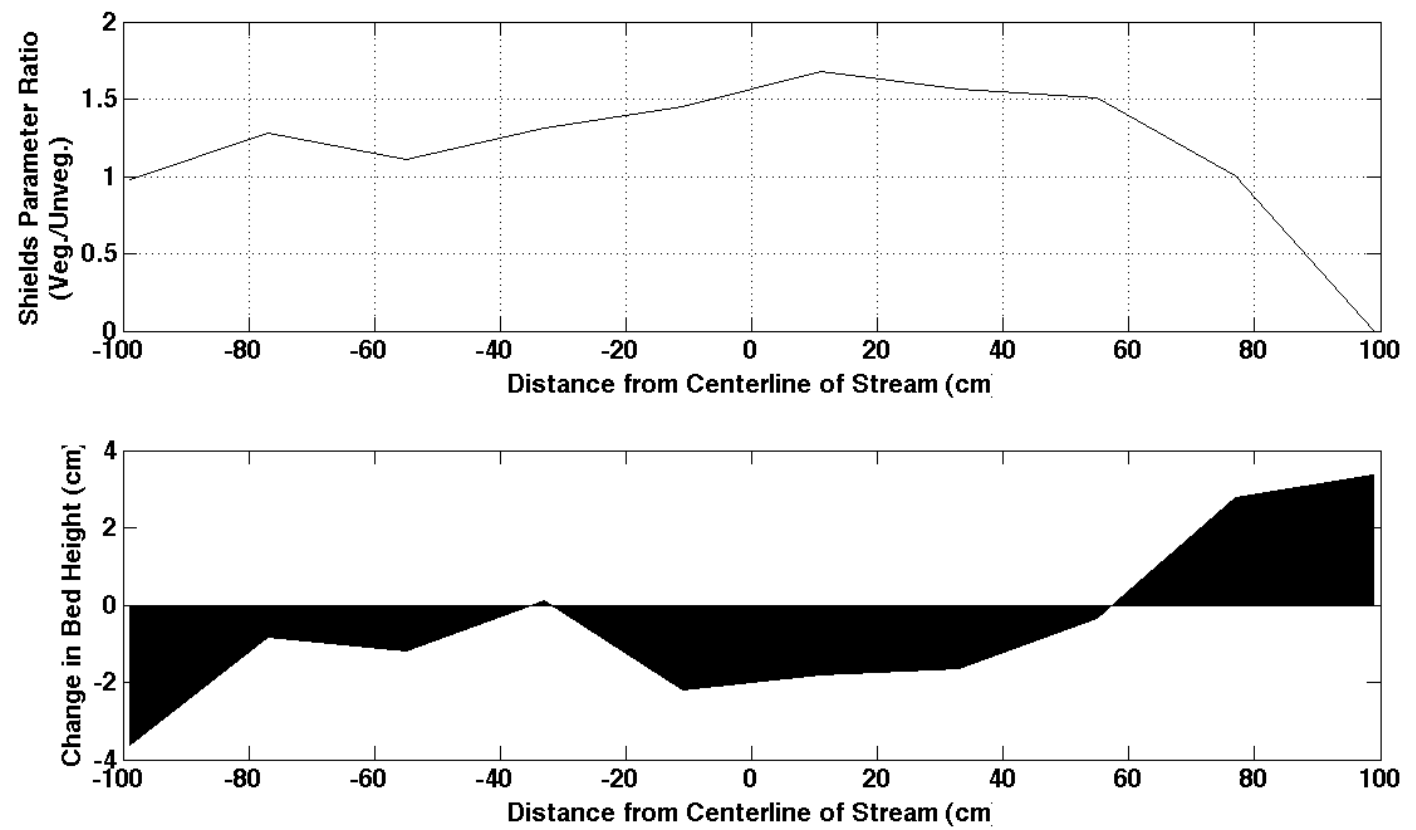

579 FIGURE 8: (a) The ratio of the Shields Parameters $\psi_{\text {veg. }} / \psi_{\text {unveg. }}$ showing the tendency of the 580 system towards either deposition $(<1)$ or erosion $(>1)$ and (b) the change in the bed height 581 following the addition of vegetation to the system. 

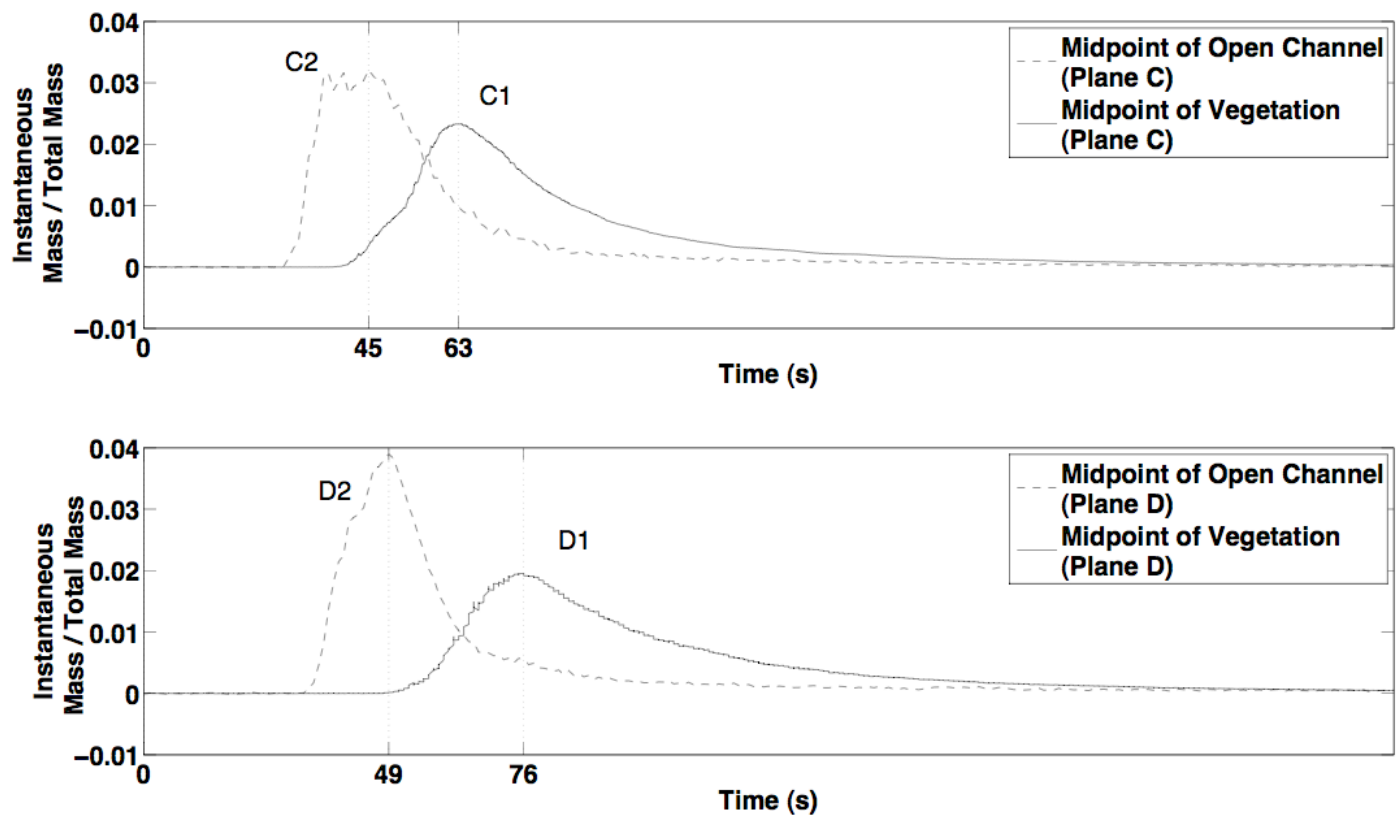

590

591

592

593

594

595

596

597

598

599

600

601

602

603

604

605

606

607

608

609

610

611
FIGURE 9: Residence time distributions of dye passing through the open channel and vegetated regions in (a) Plane C and (b) Plane D. 


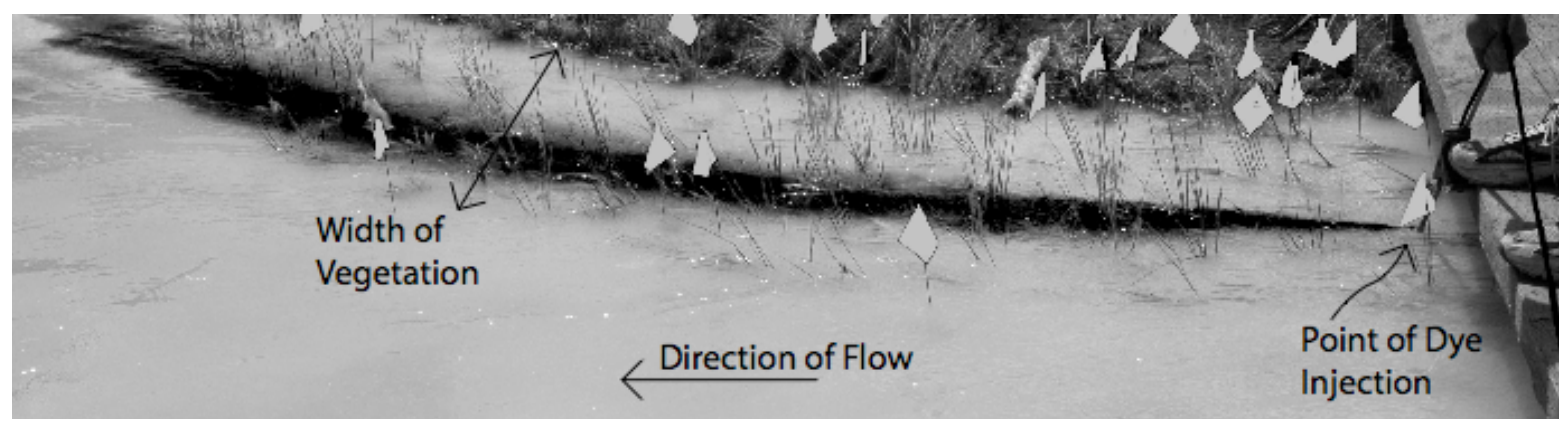

614 Figure 10: A continuous injection of Rhodamine WT (black in the exaggerated contrast image).

615 Note that the tracer cannot spread across the width of the vegetation because the observed

616 diffusion is offset by an outward advection from the bank toward the open channel. 